\title{
Role of plasminogen activator inhibitor-1 in the diagnosis and prognosis of patients with Parkinson's disease
}

\author{
HONG PAN $^{1}$, YING ZHAO ${ }^{1}$, ZHENGPING ZHAI $^{2}$, JINYU ZHENG ${ }^{3}$, YONG ZHOU ${ }^{1}$, \\ QIJIN ZHAI ${ }^{1}, \mathrm{XIANGYANG} \mathrm{CAO}^{1}$, JISHA TIAN $^{1}$ and LIANDONG ZHAO ${ }^{1}$ \\ ${ }^{1}$ Department of Neurology, Huai'an Second People's Hospital, The Affiliated Huai'an Hospital of \\ Xuzhou Medical University; ${ }^{2}$ Department of Neurology, Lianshui County People's Hospital; ${ }^{3}$ Department of \\ Neurosurgery, Huai'an Second People's Hospital, The Affiliated Huai'an Hospital of \\ Xuzhou Medical University, Huai'an, Jiangsu 223002, P.R. China
}

Received October 2, 2017; Accepted January 18, 2018

DOI: $10.3892 /$ etm.2018.6076

\begin{abstract}
Parkinson's disease is a neurodegenerative disease that frequently results in memory disorders, cognitive decline and dementia. Previous studies have reported that plasminogen activator inhibitor-1 (PAI-1) serves an important role in cardiovascular disease risk, adiposity, insulin resistance and inflammation. However, the role of PAI-1 in diagnosis and prognosis of patients with Parkinson's disease following deep brain stimulation (DBS) has not reported, to the best of our knowledge. Therefore, the purpose of the present study was to investigate the clinical significance of PAI-1 in patients with Parkinson's disease. Plasma PAI-1 levels were measured in 102 patients with Parkinson's disease who underwent DBS. It was demonstrated that plasma PAI-1 levels were significantly increased in patients with Parkinson's disease compared with healthy individuals $(\mathrm{P}<0.01)$. Patients with Parkinson's disease received DBS presented significantly improved cognitive competence compared with controls $(\mathrm{P}<0.01)$. DBS significantly decreased plasma PAI-1 levels in patients with Parkinson's disease compared with controls $(\mathrm{P}<0.05)$. It was also observed that plasma PAI-1 levels were significantly negatively associated with cognitive function for patients with Parkinson's disease $(\mathrm{P}<0.01)$. In conclusion, these findings demonstrated that the degree of Parkinson's disease severity is positively associated with circulating levels of plasma PAI-1 levels, which suggests that PAI-1 may be a potential diagnostic and prognostic marker for patients with Parkinson's disease.
\end{abstract}

Correspondence to: Professor Liandong Zhao, Department of Neurology, Huai'an Second People's Hospital, The Affiliated Huai'an Hospital of Xuzhou Medical University, 62 Nan Huahai Road, Huai'an, Jiangsu 223002, P.R. China

E-mail: zhaoliandongprof@163.com

Key words: Parkinson's disease, plasminogen activator inhibitor-1, cognitive competence, deep brain stimulation

\section{Introduction}

Parkinson's disease is a neurodegenerative disease that can lead to senile dementia and leads to higher morbidity and mortality rates among elderly populations $(1,2)$. A number of biomarkers, including dopamine transporter and aromatic L-amino acid decarboxylase for prodromal Parkinson's disease have been promising but require further study, including their application to and validation in prodromal cohorts followed longitudinally, which suggests that accurate identification of prodromal Parkinson's disease will likely require a multimodal approach (3). Pathogenesis of Parkinson's disease predominantly targets the hippocampal area, which may lead to cognitive dysfunction pertaining to memory, language or attention $(4,5)$. Previous systematic reviews and meta-analyses have demonstrated the potential diagnostic or prognostic markers that are associated with the degree of cognitive function in patients with Parkinson's disease $(6,7)$.

Plasminogen activator inhibitor-1 (PAI-1) serves an important role in the process of human cardiovascular diseases, which thereby leads to promotion of fibrinolysis $(8,9)$. Xu et al (10) have recently demonstrated that PAI-1 gene polymorphism is associated with the development and progression of predominant proteinuria diabetes nephropathy. In addition, high plasma levels of thrombomodulin, PAI-1 and fibrinogen were detected in elderly, diabetic patients with depressive symptoms and further prospective larger studies are required to provide potential directions for future research, treatment and prevention of co-morbid depression and diabetes (8). Furthermore, Zhou et al (11) have recently demonstrated that inhibition of PAI-1 activity may prevent the formation of the initial PAI-1t-PA complex, which further blocks PAI to bind to the hinge region of the reactive center loop. However, the role of PAI-1 has not been elucidated in patients with Parkinson's disease.

At present, intraoperative measurement of subthalamic nucleus width via microelectrode recording is a common proxy for optimal electrode location during deep brain stimulation (DBS) surgery for patients with Parkinson's disease (12). However, to the best of our knowledge, the role of PAI-1 in evaluating the therapeutic effects of DBS has not been investigated 
in patients with Parkinson's disease. In the present study, plasma PAI-1 levels in patients with Parkinson's disease was investigated prior to and following treatment with deep brain stimulation. The association between plasma PAI-1 levels and cognitive competence were analyzed as well as degree of Parkinson's disease. Patients with Parkinson's disease were reported to have higher plasma levels of PAI-1 and may contribute to the progression of Parkinson's disease.

\section{Materials and methods}

Study design, subjects and sampling. A total of 102 patients with Parkinson's disease (male: $n=50$, female: $n=52$ ) and 85 healthy volunteers (male: $n=42$, female: $n=43$ ) were recruited in the present study from the Affiliated Huai'an Hospital of Xuzhou Medical University between June 2013 and May 2015. The treatment period was 8 weeks and the follow-up was 36 months. The age of patients was 64.6-83.4 years. Patients were included following diagnosis of Parkinson's disease using Canadian Primary Care Sentinel Surveillance Network data (13). Staging of Parkinson's disease was performed using Hoehn-Yahr criteria (14). Patients' Positive and Negative Syndrome Scale (PANSS) was analyzed according to a previous study (15). The methodology used in the present study was approved by the Central Ethics Committee of Huai'an Second People's Hospital (Huai'an, China). Patients with cerebral hemorrhage, cerebral infarction or epilepsy were excluded. All patients were required to provide written, informed consent prior to inclusion in the present study.

Measure of blood pressure, lipids and glucose parameters. Blood pressure in patients with Parkinson's disease was measured using a non-invasive blood pressure gauge (Shanghai Yuyan Instruments Co., Ltd., Shanghai, China). The pressure value was recorded prior to and following treatment. Lipids and glucose parameters in the blood were recorded every 2 days for a total of 14 days using Amplex ${ }^{\mathrm{TM}}$ Red Glucose/Glucose Oxidase assay kit (cat. no. A22189; Thermo Fisher Scientific, Inc., Waltham, MA, USA) according to a methodology described in a previous study (16).

Montreal cognitive assessment (MoCA) score. The cognitive function of patients with Parkinson's disease was analyzed via MoCA scoring (17). Briefly, the maximal MoCA score that may be attained during analysis is 30 and a score $<26$ is considered to indicate Parkinson's disease. The maximal percent increase in each patient was calculated using the following formula: [(maximal MoCA score - MoCA score on admission)/MoCA score on admission] x100.

ELISA. Plasma levels of PAI-1 were detected prior to DBS treatment and on day 5 following DBS in patients with Parkinson's disease using an ELISA kit (cat. no. MAB32010; Bio-Techne, Minneapolis, MN, USA) according to the manufacturer's protocol.

Anxiety analysis and discrimination index. Anxiety of patients with Parkinson's disease was determined using the Quality of Recovery Score (QoR-40) as described previously (18). All patients with Parkinson's disease received DBS
Table I. Characteristics of patients with Parkinson's disease.

\begin{tabular}{lcc}
\hline Characteristic & Patients & Healthy controls \\
\hline Total, $\mathrm{n}$ & 102 & 85 \\
Male, $\mathrm{n}$ & 50 & 42 \\
Female, $\mathrm{n}$ & 52 & 43 \\
Anxiety, discrimination index & $0.73 \pm 0.08$ & $0.06 \pm 0.02$ \\
(mean \pm SD) & & \\
PANSS score (mean \pm SD) & $82.2 \pm 10.0$ & $4.6 \pm 2.2$ \\
Glucose, mmol/l (range) & $5.8-7.5$ & $5.4-7.4$ \\
Blood pressure, mmHg & $126 \pm 12$ & $118 \pm 10$ \\
(mean \pm SD) & & \\
Therapy, n & & \\
DBS & 62 & 0 \\
Placebo & 40 & 0 \\
\hline
\end{tabular}

$\mathrm{SD}$, standard deviation; PANSS, positive and negative syndrome scale.

(3.5 V; $130 \mathrm{~Hz}$; Pins Medical, Beijing, China) or placebo (control) at the subthalamic nucleus and anxiety was analyzed prior to or following this treatment according to a previously described method (19). Discrimination index was used to analyze the evidence from event-related brain potentials for patients with Parkinson's disease as described previously (20).

Regression analysis. The plasma levels of PAI-1 were subjected to regression analysis in Parkinson's disease patients at different clinical stages using least square convergence (21). The predicted curve that results in the lowest sum of squares is the best fit. If the fit is robust, the parameters of the observed curve can be inferred from those of the predicted data. All data were analyzed using SPSS software version 19.0 (IBM Corp., Armonk, NY, USA).

Statistical analysis. Data are presented as the means + or \pm the standard deviation of three repeated experiments. All data were analyzed using GraphPad Prism version 6.0 software (GraphPad Software, Inc., La Jolla, CA, USA). Unpaired data were analyzed using Student's t-test whereas comparisons of data between multiple groups were analyzed via one-way analysis of variance followed by Tukey's honest significant difference test. Kaplan-Meier analysis was used to estimate the rate of relapse and re-treatment during the 368-day treatment period. $\mathrm{P}<0.05$ was considered to indicate a statistically significant difference.

\section{Results}

Characteristics of patients with Parkinson's disease and plasma levels of PAI-1. A total of 102 patients with Parkinson's disease and 85 healthy volunteers were recruited for the present clinical analysis. All analyses were performed in an easy and comfortable environment for patients. The characteristics of patients with Parkinson's disease are summarized in Table I. It was demonstrated that the mean scores of PANSS were $82.2 \pm 10.0$. Anxiety and slow locomotion were the main external 


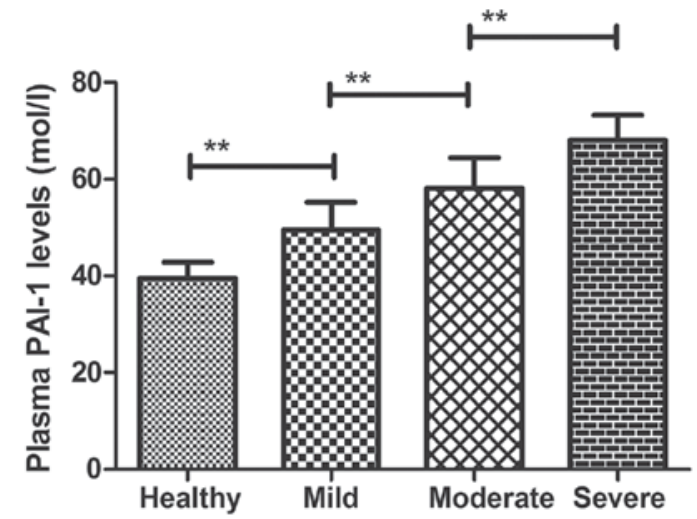

Figure 1. Plasma PAI-1 levels in patients with Parkinson's disease and healthy individuals. ${ }^{* *} \mathrm{P}<0.01$. PAI-1, plasminogen activator inhibitor-1.

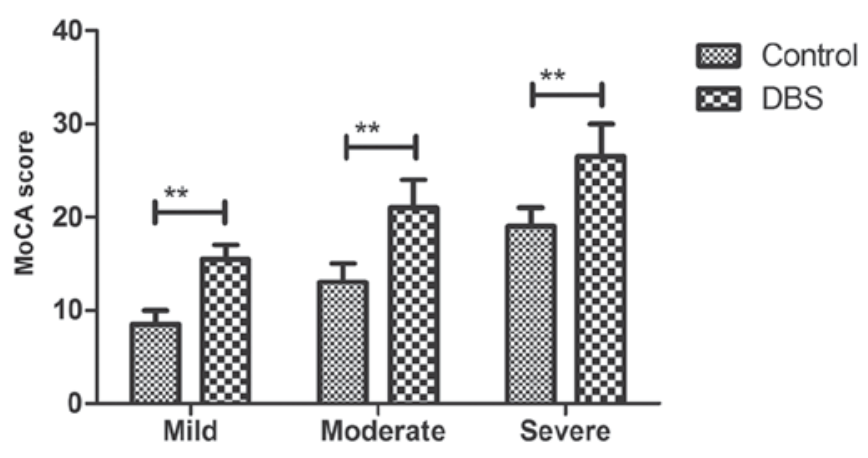

Figure 2. DBS significantly improves cognitive competence for patients with Parkinson's disease compared with control, as determined by MoCA. ${ }^{* *} \mathrm{P}<0.01$. DBS, deep brain stimulation; MoCA, Montreal cognitive assessment.

characteristics for patients with Parkinson's disease (22). Blood pressure and glucose parameters in patients were similar to healthy volunteers (data not shown). It was also demonstrated that plasma levels of PAI-1 were significantly higher in patients with Parkinson's disease compared with healthy controls (Fig. 1). Plasma levels of PAI-1 were upregulated according to severity of Parkinson's disease (Fig. 1). These results suggest that patients with Parkinson's disease present higher plasma PAI-1 levels than healthy individuals.

Efficacy of DBS for patients with Parkinson's disease. As presented in Fig. 2, patients with Parkinson's disease who received DBS presented significantly improved cognitive competence compared with controls, as determined by MoCA. It was observed that DBS treatment significantly decreased plasma PAI-1 levels in patients with Parkinson's disease (Fig. 3). It was also observed that DBS treatment significantly relieved the degree of anxiety for patients with Parkinson's disease compared with the placebo group (Fig. 4). As presented in Fig. 5, discrimination index was significantly improved by the treatment of DBS for patients with Parkinson's disease. These results suggest that DBS is beneficial for the treatment of patients with Parkinson's disease.

Association between plasma levels of PAI-1 and degree of Parkinson's disease. The association between plasma levels of PAI-1 and degree of Parkinson's disease was investigated

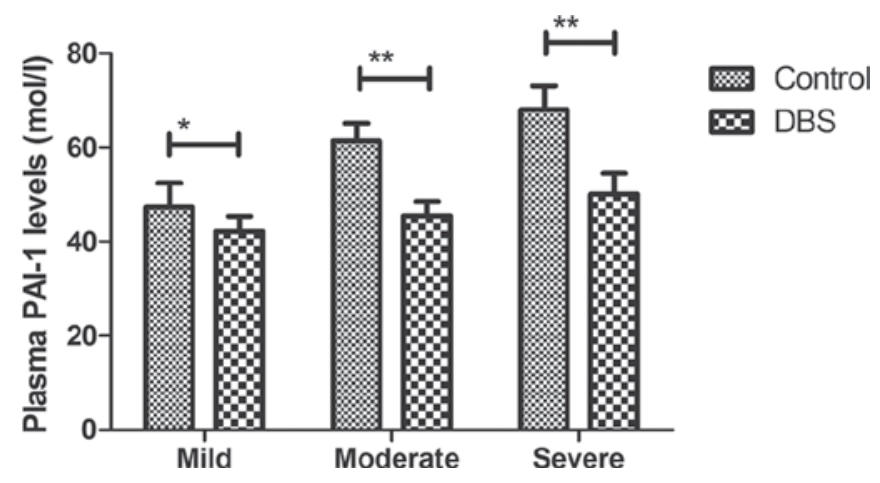

Figure 3. Deep brain stimulation treatment markedly decreases plasma PAI-1 levels in patients with Parkinson's disease. ${ }^{*} \mathrm{P}<0.05,{ }^{* *} \mathrm{P}<0.01$. PAI-1, plasminogen activator inhibitor-1; DBS, deep brain stimulation.

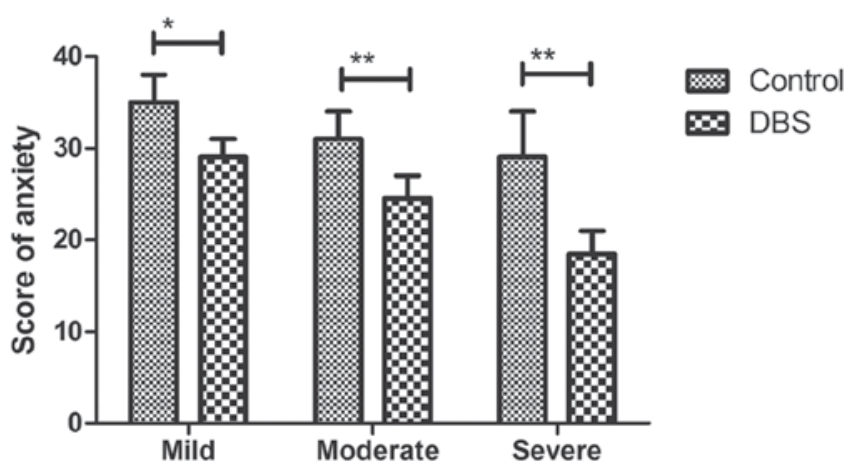

Figure 4. DBS treatment relieves the degree of anxiety for patients with Parkinson's disease compared with control group. ${ }^{*} \mathrm{P}<0.05,{ }^{* * *} \mathrm{P}<0.01$. DBS, deep brain stimulation.

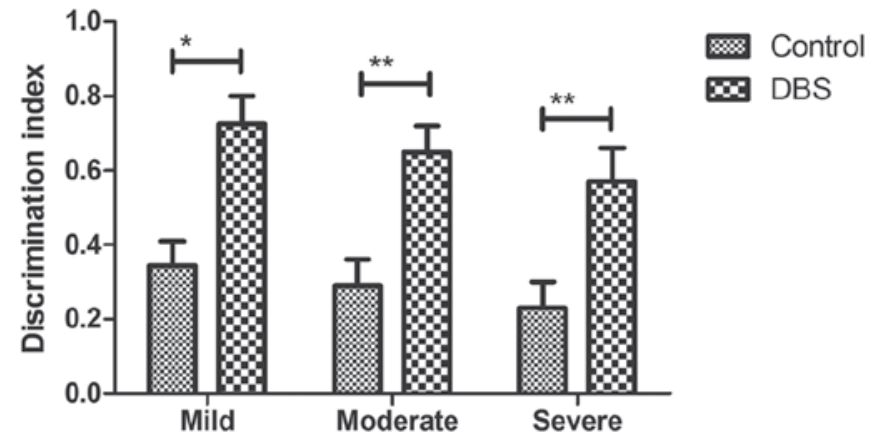

Figure 5. Discrimination index is significantly improved by the treatment of deep brain stimulation for patients with Parkinson's disease. $\mathrm{P}<0.05$, ${ }^{* *} \mathrm{P}<0.01$. DBS, deep brain stimulation.

in patients with Parkinson's disease. It was demonstrated that plasma levels of PAI-1 were a significant predictor of the degree of clinical stage of Parkinson's disease (Fig. 6). These results suggest that plasma levels of PAI-1 may be a potential biomarker for accessing the clinical stage of Parkinson's disease.

Association between plasma PAI-1 levels and cognitive function in patients with Parkinson's disease. Changes in plasma PAI-1 levels were investigated in patients with Parkinson's disease. The present results revealed that plasma 


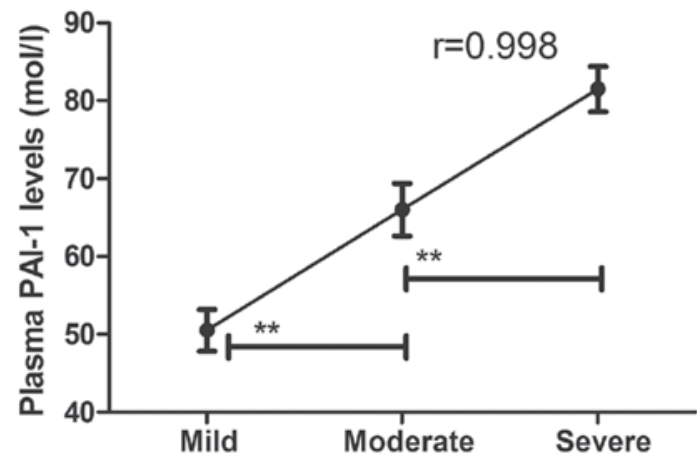

Figure 6. Plasma levels of PAI-1 are positively associated with the degree of clinical stage of Parkinson's disease. ${ }^{* *} \mathrm{P}<0.01$. PAI-1, plasminogen activator inhibitor-1.

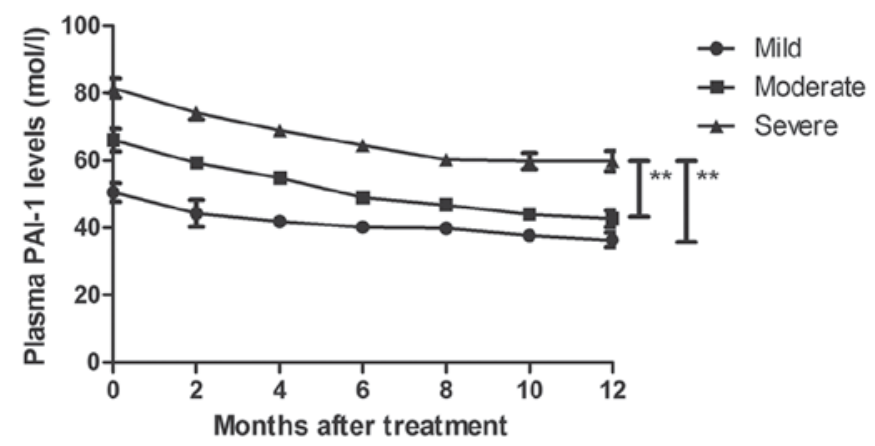

Figure 7. Plasma PAI-1 levels are decreased during the treatment of Parkinson's disease. ${ }^{* *} \mathrm{P}<0.01$. PAI-1, plasminogen activator inhibitor-1.

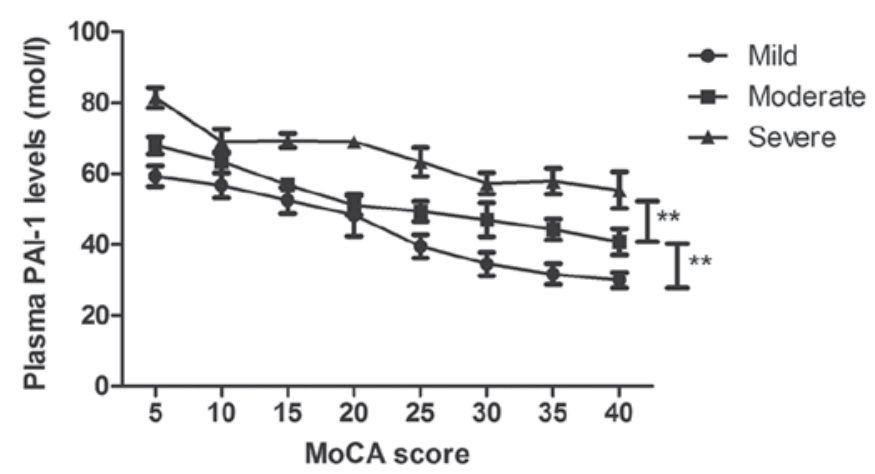

Figure 8. Plasma PAI-1 levels are negatively associated with cognitive function for patients with Parkinson's disease. ${ }^{* *} \mathrm{P}<0.01$. PAI-1, plasminogen activator inhibitor-1; MoCA, Montreal cognitive assessment.

PAI-1 levels were significantly decreased after treatment with DBS for patients with Parkinson's disease (Fig. 7). Notably, it was revealed that plasma PAI-1 levels were negatively c associated with cognitive function for patients with Parkinson's disease (Fig. 8). These results indicated that plasma levels of PAI-1 are negatively associated with cognitive function in patients with Parkinson's disease.

\section{Discussion}

Parkinson's disease is characterized by cognitive impairment and neurodegeneration $(23,24)$. Previous evidence has demonstrated that alternative treatment procedures combining drainage and intraventricular fibrinolysis with recombinant tissue plasminogen activator may prevent haemorrhagic complications (25). Notably, a previous study has reported the changes of focal and brainstem neurological signs in patients with traumatic brain injury and their dependence on the -675 4/5 G polymorphism in the PAI-1 gene (26). Therefore, in the present study it was assumed that plasma PAI-1 level may be associated with the degree of Parkinson's disease. The aim of the present study was to analyze the association between plasma PAI-1 level and cognitive function in patients with Parkinson's disease, and it was observed that plasma PAI-1 was upregulated in patients with Parkinson's disease and was positively associated with the degree of Parkinson's disease severity.

In the present study, patients with Parkinson's disease exhibited higher plasma PAI-1 levels than healthy volunteers. Although DBS of the subthalamic nucleus therapy is an effective treatment for motor impairments in Parkinson's disease (27-29), the association between PAI-1 and the efficacy of DBS have not been investigated in patients with Parkinson's disease, to the best of our knowledge. The present results support that DBS is beneficial for the treatment of patients with Parkinson's disease and revealed that plasma levels of PAI-1 are positively associated with degree of Parkinson's disease severity and negatively associated with cognitive function in patients with Parkinson's disease.

PAI-1 is abundantly expressed in infarcted myocardium, but the pathogenic role of plasma PAI-1 plasma remains unknown in patients with Parkinson's disease (30). Cho et al (31) have previously demonstrated that valproic acid may induce astrocyte-dependent neurite outgrowth from cultured rat primary cortical neuron via modulation of tissue plasmogen activator PAI-1 activity. Previous studies have reported changes in the plasminogen activator system and the inhibitors PAI-1 and PAI-2 in posttraumatic lesions in the central nervous system and brain injuries $(32,33)$. In the present study, it was observed that plasma PAI-1 levels are upregulated in patients with Parkinson's disease and DBS therapy decreased plasma PAI-1 levels, which is associated with cognitive function in patients with Parkinson's disease.

In conclusion, the present study study demonstrated that plasma PAI-1 level serves an important role in the progression of Parkinson's disease. The results indicate that DBS therapy was associated with a decrease in plasma PAI-1 levels and relieved anxiety and discrimination index in patients with Parkinson's disease. Additionally, it was observed that plasma PAI-1 levels may be a potential biomarker for assessing the clinical stage of Parkinson's disease, which is negatively associated with cognitive function in patients with Parkinson's disease. Taken together, these findings suggest that PAI-1 is a potential diagnostic or prognostic marker for patients with Parkinson's disease. However, further study is required to identify the role of plasminogen activator inhibitor-1 in the diagnosis and prognosis of patients with Parkinson's disease in a large population size.

\section{Acknowledgements}

Not applicable. 


\section{Funding}

No funding was received.

\section{Availability of data and materials}

The datasets used and/or analyzed during the current study are available from the corresponding author on reasonable request.

\section{Authors' contributions}

HP, YZ, ZZ, JZ and YZ analyzed and interpreted the patient data regarding the hematological disease and the transplant. HP, QZ, XC, JT and LZ performed the histological examination of the kidney, and HP was a major contributor in writing the manuscript. All authors read and approved the final manuscript.

\section{Ethics approval and consent to participate}

The methodology used in the present study was approved by the Central Ethics Committee of Huai'an Second People's Hospital (Huai'an, China). All patients were required to provide written, informed consent prior to inclusion in the present study.

\section{Consent for publication}

Not applicable.

\section{Competing interests}

The authors declare that they have no competing interests.

\section{References}

1. Mano T, Britton $\mathrm{Z}$ and Britton $\mathrm{T}$ : Anatomo-functional basis of nonmotor symptoms in Parkinson disease. Neurology 87: 2506-2507, 2016.

2. Meles SK, Teune LK, de Jong BM, Dierckx RA and Leenders KL: Metabolic Imaging in Parkinson disease. J Nuclear Med 58: 23-28, 2017.

3. Cooper CA and Chahine LM: Biomarkers in prodromal Parkinson disease: A qualitative review. J Int Neuropsychol Soc 22: 956-967, 2016.

4. Chiou SM, Lin YC, Lu MK and Tsai CH: Bilateral subthalamic stimulation for advanced Parkinson disease: Early experience at an Eastern center. Neurol Sci 36: 515-520, 2015.

5. Nowacka B, Lubinski W, Honczarenko K, Potemkowski A and Safranow K: Ophthalmological features of Parkinson disease. Med Sci Monit 20: 2243-2249, 2014.

6. Elstein D, Alcalay R and Zimran A: The emergence of Parkinson disease among patients with Gaucher disease. Best Pract Res Clin Endocrinol Metab 29: 249-259, 2015.

7. Liang HW, Huang YP and Pan SL: Parkinson disease and risk of acute myocardial infarction: A population-based, propensity score-matched, longitudinal follow-up study. Am Heart J 169: 508-514, 2015.

8. Gorska-Ciebiada M, Saryusz-Wolska M, Borkowska A, Ciebiada M and Loba J: Plasma levels of thrombomodulin, plasminogen activator inhibitor-1 and fibrinogen in elderly, diabetic patients with depressive symptoms. Aging Clin Exp Res 28: 843-851, 2016.

9. Gettins PG and Dolmer K: The high affinity binding site on plasminogen activator Inhibitor-1 (PAI-1) for the low density lipoprotein receptor-related protein (LRP1) is composed of four basic residues. J Biol Chem 291: 800-812, 2016.
10. Xu F, Liu H and Sun Y: Association of plasminogen activator inhibitor-1 gene polymorphism and type 2 diabetic nephropathy. Ren Fail 38: 157-162, 2016.

11. Zhou X, Hendrickx ML, Hassanzadeh-Ghassabeh G, Muyldermans S and Declerck PJ: Generation and in vitro characterisation of inhibitory nanobodies towards plasminogen activator inhibitor 1. Thromb Haemost 116: 1032-1040, 2016.

12. Shenai MB, Patel DM, Romeo A, Whisenhunt JD, Walker HC Guthrie S and Guthrie BL: The relationship of electrophysiologic subthalamic nucleus length as a predictor of outcomes in deep brain stimulation for parkinson disease. Stereotact Funct Neurosurg 95: 341-347, 2017.

13. Correction: Using canadian primary care sentinel Surveillance Network data to examine depression in patients with a diagnosis of Parkinson disease: A retrospective cohort study. CMAJ Open 4: E719, 2016.

14. Kataoka H, Tanaka N, Eng M, Saeki K, Kiriyama T, Eura N, Ikeda M, Izumi T, Kitauti T, Furiya Y, et al: Risk of falling in Parkinson's disease at the Hoehn-Yahr stage III. Eur Neurol 66: 298-304, 2011.

15. Yehya A, Ghuloum S,MahfoudZ,Opler M,Khan A,Hammoudeh S, Abdulhakam A, Al-Mujalli A, Hani Y, Elsherbiny R and Al-Amin H: Validity and reliability of the arabic version of the positive and negative syndrome scale. Psychopathology 49: 181-187, 2016.

16. Nunes PM, Wright AJ, Veltien A, van Asten JJ, Tack CJ, Jones JG and Heerschap A: Dietary lipids do not contribute to the higher hepatic triglyceride levels of fructose-compared to glucose-fed mice. FASEB J 28: 1988-1997, 2014.

17. Pelletier S, Nalpas B, Alarcon R, Rigole H and Perney P: Investigation of cognitive improvement in alcohol-dependent inpatients using the montreal cognitive assessment (MoCA) score. J Addict 2016: 1539096, 2016.

18. McIntosh S and Adams J: Anxiety and quality of recovery in day surgery: A questionnaire study using Hospital Anxiety and depression scale and quality of recovery score. Int J Nurs Pract 17: $85-92,2011$

19. Guimaraes-Pereira L, Costa M, Sousa G and Abelha F: Quality of recovery after anaesthesia measured with QoR-40: A prospective observational study. Braz J Anesthesiol 66: 369-375, 2016.

20. Wieser MJ, Klupp E, Weyers P, Pauli P, Weise D, Zeller D, Classen $\mathrm{J}$ and Mühlberger A: Reduced early visual emotion discrimination as an index of diminished emotion processing in Parkinson's disease?-Evidence from event-related brain potentials. Cortex 48: 1207-1217, 2012.

21. Hayes AF and Rockwood NJ: Regression-based statistical mediation and moderation analysis in clinical research: Observations, recommendations, and implementation. Behav Res Ther 98: 39-57, 2017

22. Kovacs M, Makkos A, Weintraut R, Karadi K, Janszky J and Kovacs N: Prevalence of anxiety among hungarian subjects with Parkinson's disease. Behav Neurol 2017: 1470149, 2017.

23. Tholfsen LK, Larsen JP, Schulz J, Tysnes OB and Gjerstad MD: Changes in insomnia subtypes in early Parkinson disease. Neurology 88: 352-358, 2017.

24. Wyman-Chick KA, Martin PK, Minár M and Schroeder RW: Cognition in patients with a clinical diagnosis of Parkinson disease and scans without evidence of dopaminergic deficit (SWEDD): 2-year follow-up. Cogn Behav Neurol 29: 190-196, 2016.

25. Leroux P and Marret S: PAI-1/t-PA ratio in cord blood: A potential index of brain haemorrhage risk in extreme preterms. Arch Dis Child Fetal Neonatal Ed 98: F281-F282, 2013.

26. Potapov O and Kmyta O: Changes of focal and brainstem neurologic signs in patients with traumatic brain injury and their dependence on the- $6754 \mathrm{G} / 5 \mathrm{G}$ polymorphism in the PAI-1 gene. Georgian Med News: 45-51, 2014 (In Russian).

27. Knight EJ, Testini P, Min HK, Gibson WS, Gorny KR, Favazza CP, Felmlee JP, Kim I, Welker KM, Clayton DA, et al: Motor and nonmotor circuitry activation induced by subthalamic nucleus deep brain stimulation in patients with parkinson disease: Intraoperative functional magnetic resonance imaging for deep brain stimulation. Mayo Clin Proc 90: 773-785, 2015.

28. Pallavaram S, D'Haese PF, Lake W, Konrad PE, Dawant BM and Neimat JS: Fully automated targeting using nonrigid image registration matches accuracy and exceeds precision of best manual approaches to subthalamic deep brain stimulation targeting in Parkinson disease. Neurosurgery 76: 756-765, 2015. 
29. Wagenbreth C, Zaehle T, Galazky I, Voges J, Guitart-Masip M, Heinze HJ and Düzel E: Deep brain stimulation of the subthalamic nucleus modulates reward processing and action selection in Parkinson patients. J Neurol 262: 1541-1547, 2015.

30. Minami Y, Horikawa K, Akiyama M and Shibata S: Restricted feeding induces daily expression of clock genes and Pai-1 mRNA in the heart of Clock mutant mice. FEBS Lett 526: 115-118, 2002

31. Cho KS, Kwon KJ, Choi CS, Jeon SJ, Kim KC, Park JH, Ko HM, Lee SH, Cheong JH, Ryu JH, et al: Valproic acid induces astrocyte-dependent neurite outgrowth from cultured rat primary cortical neuron via modulation of tPA/PAI-1 activity. Glia 61: 694-709, 2013
32. Dietzmann K, von Bossanyi P, Krause D, Wittig H, Mawrin C and Kirches E: Expression of the plasminogen activator system and the inhibitors PAI-1 and PAI-2 in posttraumatic lesions of the CNS and brain injuries following dramatic circulatory arrests: An immunohistochemical study. Pathol Res Pract 196: 15-21, 2000.

33. Zervos IA, Nikolaidis E, Lavrentiadou SN, Tsantarliotou MP Eleftheriadou EK,Papapanagiotou EP,Fletouris DJ, Georgiadis M and Taitzoglou IA: Endosulfan-induced lipid peroxidation in rat brain and its effect on t-PA and PAI-1: Ameliorating effect of vitamins $\mathrm{C}$ and E. J Toxicol Sci 36: 423-433, 2011. 